\title{
Role of the Lateral Intraparietal Area in Modulation of the Strength of Sensory-Motor Transmission for Visually Guided Movements
}

\author{
John G. O'Leary ${ }^{2,3,4}$ and Stephen G. Lisberger ${ }^{1,2,3,4}$ \\ ${ }^{1}$ Howard Hughes Medical Institute, ${ }^{2}$ Neuroscience Graduate Program, ${ }^{3}$ W. M. Keck Foundation Center for Integrative Neuroscience, and ${ }^{4}$ Department of \\ Physiology, University of California, San Francisco, San Francisco, California 94143-0444
}

\begin{abstract}
The lateral intraparietal area (LIP) has been implicated as a salience map for control of saccadic eye movements and visual attention. Here, we report evidence to link the encoding of saccades and saliency in LIP to modulation of several other sensory-motor behaviors in monkeys. In many LIP neurons, there was a significant trial-by-trial correlation between the firing rate just before a saccade and the postsaccadic or presaccadic pursuit eye velocity. Some neurons also showed trail-by-trial correlations of the firing rate of LIP neurons with the speed of "glissades" that occur at the end of saccades to stationary targets. LIP-pursuit correlations were spatially specific and were strong only when the target appeared in the receptive/movement field of the neuron under study. We suggest that LIP is a component of a salience representation that modulates the strength of visual-motor transmission for pursuit, and that may play a similar role for many movements, beyond its traditional roles in guiding saccadic eye movements and localizing attention.
\end{abstract}

\section{Introduction}

The lateral intraparietal area (LIP) seems to contain a topographic representation of the saliency of objects in the visual scene and to play an important role in motor control and perception. The representations in LIP of visual stimuli, attention, and saccadic eye movements have been studied thoroughly, but little is known about how LIP controls perception and action. In the abstract of a recent review, for example, Bisley and Goldberg (2010) conclude that "The spatial location representing the peak of the map is used by the oculomotor system to target saccades and by the visual system to guide visual attention." Their conclusion leaves open two key questions. Is there something special about saccades and LIP, or does the proposed salience map in LIP act on many forms of sensory-motor processing? If LIP modulates sensory-motor behavior more broadly, through what neural mechanisms does it act?

We have chosen to evaluate these issues through analysis of visually guided smooth pursuit eye movements. Extrastriate visual area MT provides the sensory signals that guide pursuit (Newsome et al., 1985; Groh et al., 1997). However, visual motion sensory signals do not enjoy unfettered access to the pursuit motor system. A given target motion can have quite different

\footnotetext{
Received Jan. 18, 2012; revised May 19, 2012; accepted May 24, 2012.

Author contributions: J.G.0. and S.G.L. designed research; J.G.0. performed research; J.G.0. analyzed data; J.G.0. and S.G.L. wrote the paper.

This work was supported by the Howard Hughes Medical Institute and NIH Grants MH077970, EY03878, and T32 EY007120. We are grateful to Dr. Mark Churchland for extensive and helpful comments on this paper. We also thank Drs. Michael Stryker, Christoph Schreiner, Michael Brainard, and Tirin Moore for helpful comments. We are grateful to Stefanie Tokiyama, Elizabeth Montgomery, Karen MacLeod, Dirk Kleinhesselink, Scott Ruffner, David WolfgangKimball, Darrell Floyd, and Ken McGary for technical assistance, and to Hilary Heuer for moral support.

Correspondence should be addressed to Stephen G. Lisberger, Department of Neurobiology, 101H Bryan Building, 412 Research Drive, Durham, NC 27710-0001. E-mail: lisberger@neuro.duke.edu.

DOI:10.1523/JNEUROSCI.0269-12.2012

Copyright $\odot 2012$ the authors $\quad 0270-6474 / 12 / 329745-10 \$ 15.00 / 0$
}

effects on pursuit depending on the state of the motor system (Schwartz and Lisberger, 1994; Churchland et al., 2003) and the intention of the subject. If given proper instructions, pursuit can become preferentially or selectively responsive to the visual motion inputs from a specific target when multiple motions are present (Gardner and Lisberger, 2002; Carello and Krauzlis, 2004; Krauzlis, 2004; Garbutt and Lisberger, 2006; Shichinohe et al., 2009). These functions involve spatially specific control of the strength of visual-motor transmission (Schoppik and Lisberger, 2006) mediated in part by the smooth eye movement region of the frontal eye fields (Tanaka and Lisberger, 2001).

In many ways, the modulation of the strength of visualmotor transmission for pursuit fits with the expected action of a salience map in LIP. Saccades provide one of the most powerful ways to modulate the strength of visual-motor transmission (Lisberger, 1998; Carello and Krauzlis, 2004; Schoppik and Lisberger, 2006). The map in LIP would be highly active just before a saccade, and the most active neurons would represent the endpoint of the saccade. Perhaps LIP modulates the sensory transmission for pursuit and other movements, with an especially strong effect on motor behaviors that are coordinated with saccades.

We have taken advantage of the natural variation in the responses of LIP neurons while monkeys used saccades and pursuit to track smooth target motion. We found correlations between the trial-bytrial fluctuations in the activity of LIP neurons and the eye velocity of postsaccadic pursuit. The timing of the correlations suggests that the salience map reflected in LIP modulates the strength of visual-motor transmission for pursuit. The use of spatially specific modulation of the gain of visual-motor transmission as the efferent mechanism of a salience map suggests a mechanism by which LIP could control many sensory-motor transformations. 


\section{Materials and Methods}

We recorded eye movements and neural activity from two adult male rhesus monkeys (Macaca mulatta). We used surgical procedures that have been described previously (Ramachandran and Lisberger, 2005) to instrument each monkey with a socket for head restraint and a scleral search coil for monitoring eye movements. In a separate surgery, we implanted a titanium recording cylinder that was placed normal to the skull over a craniotomy centered at stereotaxic coordinates: P5, L12. Before the experiments described here, the monkeys had been trained to sit in a primate chair with their heads restrained, and to fixate and track targets presented on a video display in exchange for liquid reinforcement. All procedures were approved in advance by the Institutional Animal Care and Use Committee at University of California, San Francisco, and were in compliance with the National Institutes of Health Guide for the Care and Use of Laboratory Animals.

Behavioral procedures. The presentation of visual stimuli and the acquisition of behavioral data were controlled by a real-time application developed in our laboratory. The software ran on a PC under Windows XP and used the real-time kernel RTX (VentureCom). The Maestro PC sent commands for the display of the visual stimuli over the local area network to a Linux PC that controlled a monitor to present visual stimuli to the monkey. For monkey I, stimuli were presented on a 20 inch, $1280 \times 1024$ resolution, CRT monitor that was positioned $38 \mathrm{~cm}$ from the monkey's eyes, yielding a field of view of $56 \times 43^{\circ}$. For monkey $\mathrm{G}$, the resolution of a 22 inch CRT was $2304 \times 1440$ pixels, the viewing distance was $41 \mathrm{~cm}$, and the field of view was $60 \times 40^{\circ}$. The temporal refresh rate of the monitors was $85 \mathrm{~Hz}$.

The horizontal and vertical eye position signals generated by the scleral search coil were differentiated by analog circuits that rejected frequencies $>25 \mathrm{~Hz}$ with a roll-off of $20 \mathrm{~dB}$ per decade. Eye position and velocity signals were sampled at $1 \mathrm{kHz}$ on each channel and saved to disk.

Electrophysiological procedures. In each daily experiment, up to five platinum/tungsten electrodes (impedance, 2-4 M $\Omega$ ) were lowered into the recording cylinder with a Mini Matrix microdrive (Thomas Recording). Neural signals were amplified and filtered conventionally, and a real-time template-matching system was used to select waveforms of single units (Plexon). For most units, a raw spike waveform was digitized, and isolation was checked and improved off-line using custom software. For the remaining units, the Plexon off-line sorter was used instead.

We classified units as located in area LIP if they had restricted visual receptive fields (Ben Hamed et al., 2001) and displayed the characteristic memory or saccadic period activity patterns of cells in that area during the memory saccade task (Barash et al., 1991). We also included neurons that were found near an abundance of other neurons that displayed those characteristic activities.

For receptive field mapping, the monkey fixated a dim red point while a bright white $1^{\circ}$ spot was flashed at different locations in the visual field. In each behavioral trial, we presented eight repetitions of a $200 \mathrm{~ms}$ flash followed by a $500 \mathrm{~ms}$ interval between presentations. The mapping procedure involved 48 different stimulus locations, each presented at least six times. We counted the spikes from the neuron under study during each $200 \mathrm{~ms}$ stimulus presentation, averaged across the six or more stimulus presentations, and then interpolated a smooth receptive field surface. After estimating the location of the receptive field with coarsely spaced stimulus locations that covered much of the monitor, we reduced the spacing between the points on the grid, shifted the center of the grid, and remapped at a higher resolution.

Once we had estimated the center of the receptive field of the neuron, we measured the responses of the neuron during a standard memoryguided saccade task (Barash et al., 1991). Trials began with fixation of a stationary spot at the center of the screen for 700-1200 ms. Then, the ultimate saccade target appeared for $200 \mathrm{~ms}$. After a memory period of random duration between 800 and $1200 \mathrm{~ms}$, the fixation spot disappeared and the monkey had $500 \mathrm{~ms}$ to make a saccade to the remembered location of the saccade target. For each neuron, we recorded the responses for memory-guided saccades for two target locations, in separate but interleaved trials. One target location was in the center of the recep-

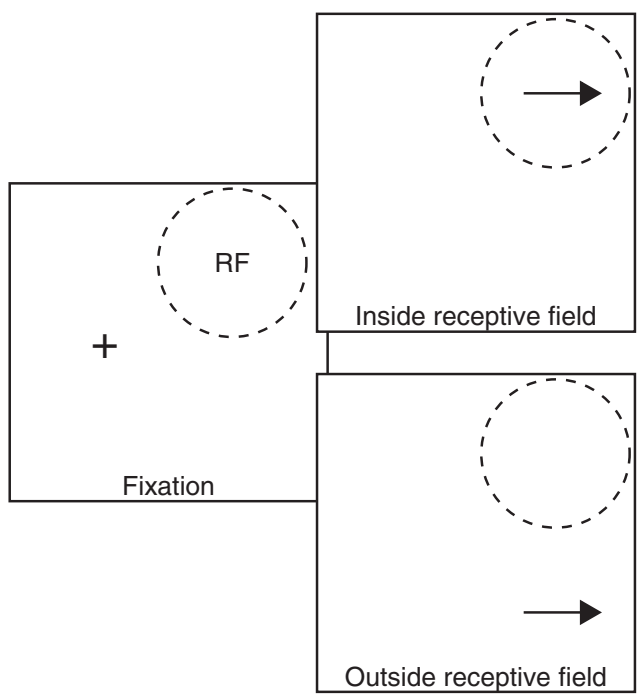

Figure 1. Schematic diagram showing stimulus configuration for pursuit trials. The cross in the left panel represents the fixation point, and the dashed circle represents the receptive field of the neuron under study. The top and bottom right panels show a target appearing inside or outside the receptive field. The arrow indicates the direction of target motion.

tive field, and the other location well outside the receptive field of the neuron under study.

Experimental paradigm. Pursuit trials began when monkeys fixated within $2^{\circ}$ of a central target for a random duration of $800-1400 \mathrm{~ms}$. The fixation point then disappeared and a moveable target appeared and immediately moved either leftward or rightward for 500-600 ms. The target then stepped $1^{\circ}$ further in the direction of motion and became a stationary fixation target for a random period of 500-600 ms. The additional fixation period at the end of the trial helped to improve monkeys' tracking performance throughout the trial. After the pursuit target appeared and started to move, the monkeys were given a grace period of 400 $\mathrm{ms}$ to bring their eyes within a fixation window of $3^{\circ}$ (monkey I) or $4^{\circ}$ (monkey G) around the moving target. They received rewards for keeping eye position within the window around the target for the duration of the trial.

Figure 1 shows an example of how target positions and motion directions were chosen. Most experiments contained two trial types with pursuit targets that appeared with equal likelihood in random order either inside or well outside the receptive field of the neuron under study. A few early experiments in monkey I did not contain targets outside the receptive field. We recorded 200-250 repetitions of each trial in monkey I, and $300-450$ trials in monkey G. After a satisfactory number of trials had been collected during the first block, we ran one or two additional blocks using different initial target positions. Control analyses revealed that the exact location of the target inside the receptive field did not influence the effects we report here, so we have elected to ignore minor differences in target placement between the two monkeys and across neurons. The direction of target motion was either leftward or rightward and was chosen so that (1) motion always was toward the center of the receptive field, toward regions that produced larger responses during receptive field testing; and (2) presaccadic pursuit was minimized and the temporal reliability of the first saccade toward the target was maximized. In general, the second constraint dictated that the target moved at least somewhat away from the position of fixation. After we completed blocks with pursuit trials, for some neurons in monkey G, we collected one or two blocks of data using a visually guided saccade task that had the same stimulus geometry and timing as the pursuit task, but with stationary rather than moving targets. Our analysis of trial-by-trial correlations between LIP activity and glissades was performed using data from visually guided saccades, rather than memory-guided saccades.

We designed the presentation of target motions in a way that would maximize the amount of useful data collected in the shortest possible time, creating the situation in which the number of experiments varied 
across neurons. For a given neuron, we might complete only a single experiment with moving targets, multiple experiments with moving targets, or multiple experiments with moving targets and one or two additional experiments that used stationary targets. Each experiment comprised a set of data with enough controls to allow conclusions, but an individual neuron might contribute as few as one or as many as five experiments. We will structure our data presentation in terms of experiments, recognizing that some experiments are not truly independent of others that might have been conducted on the same neuron. We have verified that the nonindependence of some of our data sets did not inflate our findings about the strength of neuron-behavior correlations for LIP neurons.

Data analysis. For each trial, software computed estimates of saccade onset and offset from the eye velocity traces by finding intervals in which the eye speed exceeded $50 \%$ s and expanding the intervals on each side by fixed offsets. We then viewed the data on a video screen and refined the marks for saccade onset and offset as necessary. At this time, some trials were discarded either because of slow eye movements indicative of sleepiness, or because of ambiguity in the end time for the first saccade after the onset of target motion. On average, $5.6 \pm 6.6 \%(\mathrm{SD})$ of trials were rejected from each experimental block.

Most of our analyses involved Pearson's correlation and partial correlation coefficients. Pearson's partial correlation coefficient $r_{x y . z}$ gives the strength of the linear relationship between two variables, $x$ and $y$, after the effect of a set $\mathbf{Z}$ of additional variables has been removed. This quantity can be written in terms of the partial correlations of progressively smaller sets of variables as follows:

$$
r_{x y . \mathbf{Z}}=\frac{r_{x y . \mathbf{Z}\{\{z\}}-r_{x z . \mathbf{Z}\{z\}} r_{y z . \mathbf{Z}\{z\}}}{\sqrt{1-r_{x z . \mathbf{Z}\{\{z\}}^{2}} \sqrt{1-r_{y z . \mathbf{Z} \backslash\{z\}}^{2}}},
$$

where $\mathbf{Z} \backslash\{z\}$ denotes the set of all variables in $\mathbf{Z}$ except $z$. The recursive solution converges when $\mathbf{Z}$ contains only $z$ as follows:

$$
r_{x y . \mathrm{Z}}=\frac{r_{x y .}-r_{x z} r_{y z}}{\sqrt{1-r_{x z}^{2}} \sqrt{1-r_{y z}^{2}}}
$$

Partial correlations can also be computed by correlating the residuals of the regression of $x$ on $\mathbf{Z}$ with the residuals of the regression of $y$ on $\mathbf{Z}$, which is what we did in practice with the MATLAB function partialcorr. The following $t$ statistic is defined for a partial correlation coefficient as follows:

$$
t=r_{x y . \mathrm{Z}} \sqrt{\frac{n-2-k}{1-r_{x y . \mathrm{Z}}^{2}}},
$$

where $k$ is the number of variables in $\mathbf{Z}$. If all the variables are normally distributed, this statistic is distributed as $t_{n-2-k}$ under the null hypothesis of no relationship between $x$ and $y$ when $\mathbf{Z}$ is controlled for. We used this $t$ statistic as an initial threshold when we tested for significant stretches of partial correlation coefficients, as described in more detail at the relevant location in Results. To ensure that our correlations were not affected by the linearity assumption of the Pearson correlation, we repeated the analysis with the Spearman partial correlation and confirmed that the results were essentially the same.

We also measured the parameters of saccadic eye movements. These included the following: latency, measured as the time from target onset to hand-marked saccade; amplitude, measured as the difference between eye position at saccade onset and hand-marked saccade offset; duration, measured as the time difference between saccade onset and offset; and peak speed, after digital differentiation using a causal filter with a $25 \mathrm{~Hz}$ cutoff.

\section{Results}

We recorded the activity of 80 neurons in area LIP (34 from monkey I; 46 from monkey G) while they performed a task that required them to make a saccade to and then pursue a target that moved through or outside the receptive field of the neuron under study. Prior research has shown that the smooth eye velocity after a saccade is enhanced as a consequence of the execution of the saccade (Lisberger, 1998; Gardner and Lisberger, 2001, 2002). Our goal was to ask whether LIP might be involved in postsaccadic enhancement of pursuit. Our approach was to analyze the trial-by-trial correlations between the activity of LIP neurons and the eye velocity after the first saccade to the moving target.

\section{Eye movements and responses of a typical LIP neuron}

The target motions we used to record from LIP neurons comprised a change in target position from the point of fixation into the receptive field of the neuron under study, followed by a ramp motion at constant speed that took the target away from the position of fixation (Fig. $2 \mathrm{~A}$, dashed trace). The monkey fixated the target before it was displaced, and then showed a latency of almost $200 \mathrm{~ms}$ before a saccade that brought the eye close to the moving target. Immediately after the saccade, the eye moved smoothly and tracked the target quite closely. Eye velocity (Fig. $2 C$ ) showed a very small change before the first saccade, a rapid and large deflection in relation to the saccade, and then substantial postsaccadic eye velocity. The gray traces in Figure 2, $A$ and $C$, illustrate considerable trial-by-trial variation in saccade latency and the magnitude of postsaccadic smooth eye velocity. Our analysis will capitalize on that variation.

LIP neurons responded during the smooth pursuit task in a manner that made sense given their receptive fields and perisaccadic responses. When the pursuit target appeared inside the receptive field of the example neuron in Figure $2 B$ and started to move, the cell fired an initial burst of spikes $\sim 50 \mathrm{~ms}$ after target onset, and then paused briefly before emitting a longer period of spikes that reached firing rates $>100$ spikes/s. The neuron returned to a low level of activity soon after the onset of a saccade from the fixation point to the target (red dots in the rasters). When the target appeared outside the receptive field (Fig. 2D), the neuron displayed a small transient increase in firing rate in response to the visual stimulus followed by a long dip to almost zero firing after a saccade to the moving target. As with eye velocity, the rasters in Figure 2, $B$ and $D$, indicate that there was considerable trial-by-trial variation in the firing of LIP neurons.

The LIP neurons in our sample responded to the saccadic eye movements that were used to bring the eye to the moving tracking target. The average firing rate profiles of individual neurons (Fig. $2 E, F$, gray traces) generally rose to a peak that was reached before the end of the saccade. The trajectories of firing rate were similar for LIP neurons recorded in the two monkeys for moving targets that appeared in the receptive field (Fig. 2E,F), as well as for stationary targets that appeared in the receptive field in monkey $\mathrm{G}$ (Fig. 2G). If anything, the responses to saccades to a stationary target were longer in duration than responses for saccades to moving targets. The LIP neurons emitted essentially no response when the target appeared outside the receptive field (Fig. $2 E, F$, red traces). We studied a few neurons for targets that ramped in two opposite directions after appearing in the receptive field and did not see evidence of strong direction selectivity for target motion, in agreement with the data of Kusunoki et al. (2000).

\section{LIP-pursuit correlations for postsaccadic eye velocity}

Our analysis capitalizes on the trial-by-trial variation in both the neural activity and the eye velocity within each experimental block. To evaluate the trial-by-trial covariation between neural activity in LIP and pursuit speed, we computed partial correlation coefficients $r_{\text {LIP,E }}^{\prime}$, where LIP denotes a smooth, time-varying estimate of firing rate created by convolving single trial spike 
trains with a Gaussian of $\sigma=40 \mathrm{~ms}$ and $\dot{E}$ is the time-varying pursuit speed. We obtained the same results, but with some extra noise, when we computed firing rate with $\sigma=20 \mathrm{~ms}$ or $\sigma=10 \mathrm{~ms}$.

The value of $r_{\mathrm{LIP}, \dot{E}}^{\prime}$ represents the linear relationship that remains between LIP and $\dot{E}$ after the correlations with saccade latency, saccade amplitude, saccade duration, and peak saccadic speed have been removed. Before performing the correlation analysis, we aligned spike trains and behavioral data with respect to the end of the saccade (Fig. 3A). We calculated the LIP-pursuit correlation for each combination of time in the firing rate across the trial and time in the first 100 postsaccadic milliseconds of the eye velocity behavior. To visualize the data, we plotted each value of $r$ in pseudocolor at the appropriate pixel for each combination of times (Fig. 3C-E).

The color surface in Figure $3 C$ has a horizontal red stripe indicating a wide swath of positive values of $r_{\text {LIP, }}^{\prime}$ for an example LIP neuron studied with pursuit of a target that moved across the receptive field of the neuron. LIP-pursuit correlations were similar when the analysis was performed for firing rate across the interval from 50 to $225 \mathrm{~ms}$ before the end of the saccade, and eye velocity from 0 to $100 \mathrm{~ms}$ after the end of the saccade. Because $r_{\text {LIP, }}^{\prime}$ was fairly constant across the first $100 \mathrm{~ms}$ of postsaccadic eye velocity, we chose to perform subsequent analyses on the eye velocity value obtained by averaging the eye speed on each trial between 20 and 60 $\mathrm{ms}$ after saccade end. We chose this interval because it was immune to any residual eye velocity from the saccade itself and preceded the first effects of visual feedback on pursuit (Lisberger, 1998). We then computed the correlation of the single measure of postsaccadic eye velocity with firing rate at each time up to the end of the saccade, which we defined as time 0 . In the bold curve in Figure $3 B$, for example, the LIP-pursuit correlation rose to a positive peak of $\sim 0.26$ for neural activity $\sim 100 \mathrm{~ms}$ before the end of the saccade. The lower bounds of bootstrapped 95\% confidence intervals for the LIPpursuit partial correlations (gray ribbon) exceed zero for firing rates from $\sim 150$ to $50 \mathrm{~ms}$ before the end of the saccade.

Figure $3 E$ also reveals an impressive swatch of correlation for firing rates $\sim 250 \mathrm{~ms}$ before the end of the saccade and eye velocity 60-100 ms after the end of the saccade. This unexpected correlation appeared only for monkey $G$ (Fig. $3 E$ ), but not for monkey I (Fig. 3D). We do not have any explanation for the correlation, and we expect that any explanation would be complicated because the eye velocity interval falls outside the times when eye velocity would be driven only by the image motion present before the saccade.

\section{Population data for LIP-pursuit correlations}

The LIP-pursuit correlations were similar for the two monkeys (Figs. 3D,E, 4A). These images and curves were obtained by
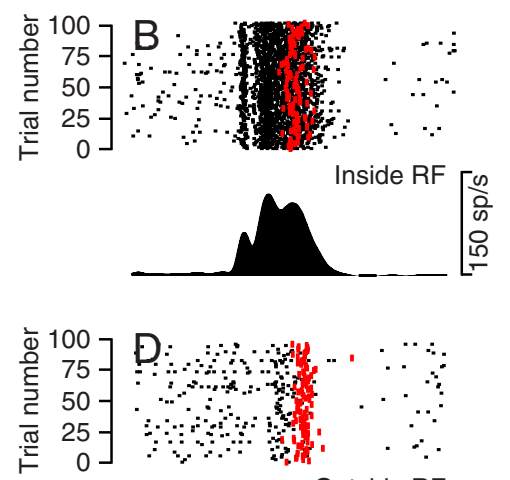

Outside RF

\section{$\left[\begin{array}{l}\infty \\ \text { क } \\ 0 \\ 10\end{array}\right.$}

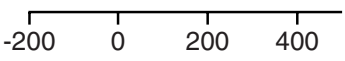

Time from target motion onset (ms)

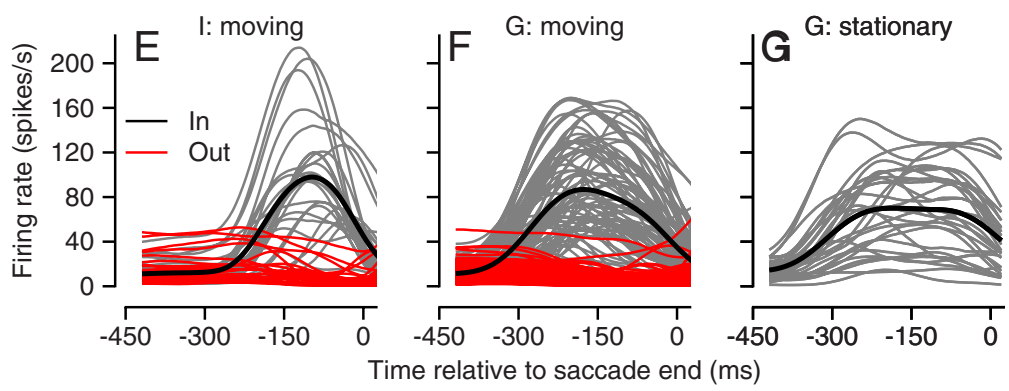

Time relative to saccade end $(\mathrm{ms})$

Figure 2. Eye movements and neural activity from a representative experiment and summaries of average neural responses of ple population. $\boldsymbol{A}, \boldsymbol{C}$, Continuous and dashed traces show eye and target position $(\boldsymbol{A})$ and velocity $(\boldsymbol{C})$ along the direction of 列

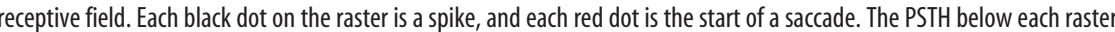

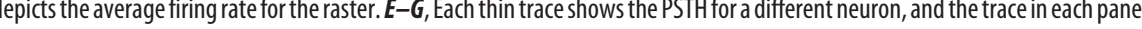
task for monkey I. $\boldsymbol{F}$, Pursuit task for monkey G. G, Saccade task for monkey G. In $\boldsymbol{E}$ and $\boldsymbol{F}$, the black and red traces show averages of firing rate for targets that appeared inside versus outside the receptive field of the neuron under study.

averaging the LIP-pursuit correlations across multiple experiments in each monkey. Recall that time on the $x$-axis in Figure $4 \mathrm{~A}$ indicates the time during the firing rate trace and that the correlation is between firing rate at that time and the mean eye velocity averaged across the fixed interval from 20 to $60 \mathrm{~ms}$ after the end of the saccade, following the analysis procedure for postsaccadic smooth eye velocity validated by Lisberger (1998). In both monkeys, the LIP-pursuit correlations peaked at a value of $\sim 0.06$ for firing rates measured $\sim 50 \mathrm{~ms}$ before the end of the saccade. The SEs (Fig. $4 A$, gray ribbons) remained well above zero for $\sim 50 \mathrm{~ms}$ on each side of the peak correlation, indicating that the average LIP-pursuit correlation at these times was reliable. To maximize the size of our data set, some neurons contributed more than one experiment using targets at slightly different positions in the receptive field. To verify that we had not inflated the LIP-pursuit by combining different numbers of conditions from different neurons, we repeated the analyses in Figure 4 by computing one LIP-pursuit correlation for each neuron from all trials at all target positions in the receptive field. The two analyses yielded the same results.

Positive LIP-pursuit correlations were present on average in both monkeys when the target appeared inside the receptive field of the neuron under study (Fig. $4 B, C$, continuous curves with 

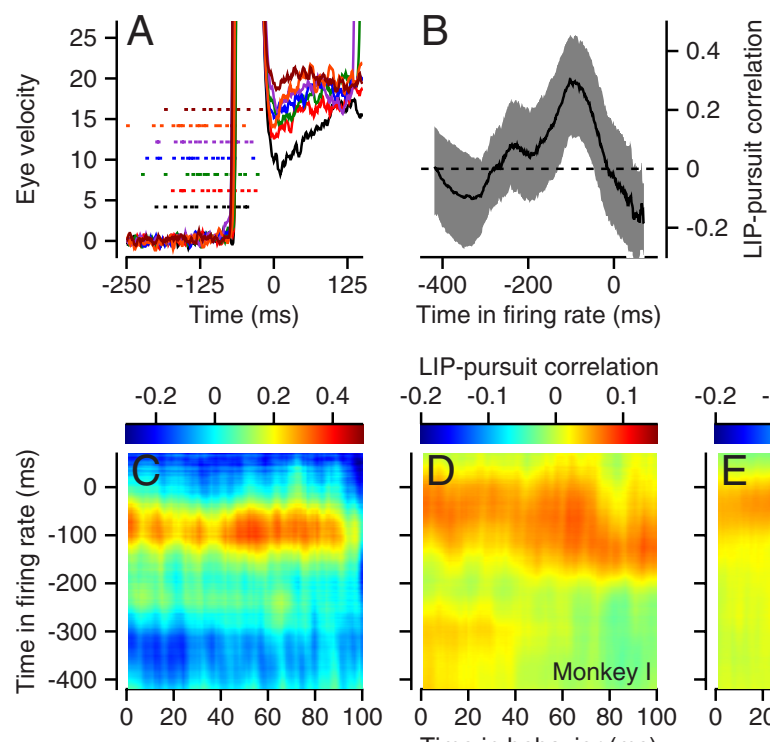

LIP-pursuit correlation

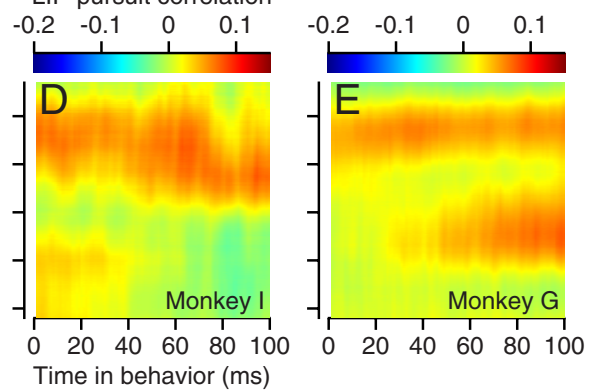

Figure 3. LIP-pursuit correlations for an example experiment and across experiments. $A$, Traces show eye velocities from single trials and dots are the spike times for the same trials. The colors indicate behavioral and neural data taken at the same time. $\boldsymbol{B}$, The bold trace is the correlation between neural activity at the time indicated on the $x$-axis and the average postsaccadic pursuit speed from 20 to $60 \mathrm{~ms}$ after the end of the saccade. The surrounding gray ribbon shows the bootstrapped $95 \%$ confidence limits. C, The color of each pixel in the image gives the LIP-pursuit partial correlation for firing rate and eye velocity for the pair of times indicated by the $x$ - and $y$-coordinates of the pixel. $\boldsymbol{D}, \boldsymbol{E}$, Average LIP-pursuit correlation maps across neurons in each monkey, configured as described for $\boldsymbol{C}$.
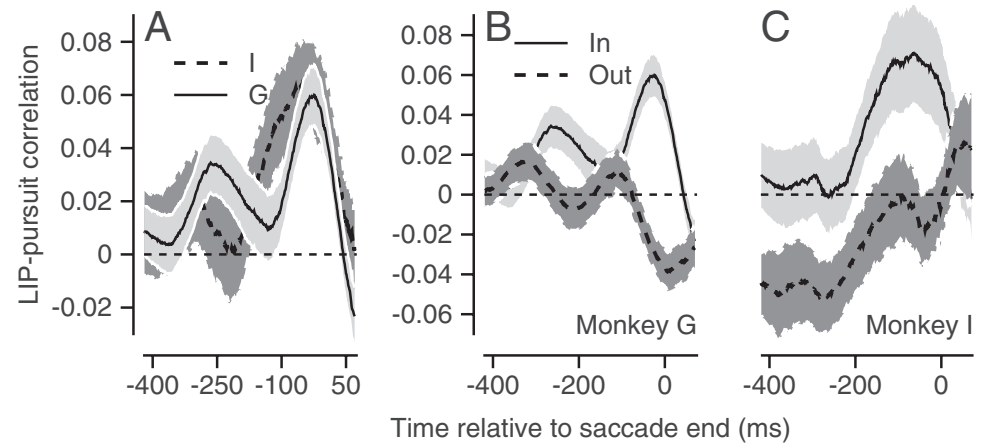

Figure 4. Summary of LIP-pursuit correlations for all experiments. $A$, The average LIP-pursuit correlations for each monkey as a function of the time of the neural activity used for the correlation. The light and dark gray show data from monkeys $G$ and I. B, C, The solid and dashed curves show the time averages of LIP-pursuit correlations for stimuli that appeared inside or outside the receptive field. The two graphs show data for the two monkeys. All error ribbons represent the SEM. The data for monkey $G$ are identical in $\boldsymbol{A}$ and $\boldsymbol{B}$, but appear different because of the different scales on the $y$-axis. The data for monkey I are slightly different in $\boldsymbol{C}$ versus $\boldsymbol{A}$ because we excluded a few experiments from $\boldsymbol{C}$ that did not include targets presented outside the receptive field.

light gray ribbons) and were absent on average when the target appeared outside the receptive field (dashed curves with dark gray ribbons). In Figure $4 B$, monkey $\mathrm{G}$ had a small positive peak in LIP-pursuit correlation $\sim 250 \mathrm{~ms}$ before the end of the saccade. We do not have an explanation for this peak, although it is possible that it results from weak correlations in the responses of LIP neurons across the time of a trial.

The average LIP-pursuit correlations showed small negative troughs for both monkeys when the target was outside the receptive field, but the timing of the troughs differed for the two monkeys. The trough occurred well before saccade onset for monkey I and near saccade onset for monkey G, at the same time as the significant positive correlation for targets in the receptive field of the neuron under study. Because we did not observe similar time courses in the two monkeys for LIP-pursuit correlations for tar- gets outside the receptive field, we are reluctant to interpret the negative correlations strongly. The long interval of complete separation of the error ribbons for targets inside versus outside the receptive field in both monkeys persuades us of the statistical significance of the spatial effect, at least for firing rate around time 0 , near the onset of the saccade.

The average time courses of LIP-pursuit correlations reveal important trends that were present in both monkeys, but also mask considerable variation in correlation strength across experiments. As illustrated by the scatter plot in Figure 5, the LIP-pursuit correlations were much stronger in some experiments (and some neurons) than in others. The strength of LIP-pursuit correlations was positively correlated with the average firing rate of the neuron at the time used to compute LIP-pursuit correlations: peak firing rate explained 13.6 and $14 \%$ of the variance in average LIP-pursuit correlation for monkeys G and I. Similar analyses failed to reveal a convincing relationship between LIP-pursuit correlation and either the position of the target within the receptive field or the features of the responses of each neuron during the memory saccade task.

The relationship in Figure 5 might indicate that LIP-pursuit correlations are by necessity weaker when firing rate is lower, or could indicate that neurons with larger responses also tend to be more strongly involved in the behavior. Given this uncertainty, the absence of positive LIP-pursuit correlations for targets that appeared outside the receptive field may indicate that neurons have a strong effect on behavior only when the target is in their receptive field.

\section{Statistical reliability of \\ LIP-pursuit correlations}

We used a suprathreshold cluster test (Nichols and Holmes, 2002) to test whether the LIP-pursuit correlations were significantly larger than zero. The suprathreshold cluster test asks whether any temporal stretches of "high" correlation in the data are longer than would be expected based on chance if there were no genuine relationship between neural activity and behavior. A test that looks at temporal sequences controls the familywise error rate and thereby avoids the multiple testing problem that arises when testing the significance of correlation coefficients that evolve across time, as seems to be the case for LIP-pursuit correlations.

We performed the cluster test separately for each experiment using targets that moved through the receptive field of the LIP neuron under study. First, we created a time sequence of the one-tailed $t$ statistic for $r_{\mathrm{LIP}, \dot{E}}^{\prime}$ at every time point. We used a onetailed test because we are testing the null hypothesis that the maximum cluster length is not $>0$. Following procedures com- 
monly used in the fMRI literature (Nichols and Holmes, 2002), we identified the longest "cluster" of contiguous time points with a $t$ statistic that exceeded a threshold of 2 (approximately equivalent to $p<0.025$; see below), and defined the length of the "maximum cluster" as $L_{\max }$. The cumulative distribution of $L_{\max }$ for each monkey (Fig. 6A) reveals that monkey G's experiments generally produced longer maximum clusters than did monkey I's. Approximately 42 versus $24 \%$ of experiments contained significant clusters of LIP-pursuit correlation that were $>50 \mathrm{~ms}$ in monkey $\mathrm{G}$ and I, respectively.

Next, to provide a level against which to test the significance of the cluster length for the LIP-pursuit correlations, we permuted the relationship between the single-trial postsaccadic velocities and firing rate profiles randomly 1000 times and computed $L_{\max }$ for each synthetic dataset. We concluded that experiments had significantly positive LIP-pursuit correlations if $<5 \%$ of the synthetic datasets yielded values of $L_{\max }$ that were equal in length or longer than the true $L_{\max }$. For the experiment illustrated in Figure $6 B, 77.5 \%$ of the synthetic datasets had clusters with $L_{\max }$ of one, while the actual $L_{\max }$ (dashed line) of 161 exceeded the $L_{\max }$ predicted by $99.7 \%$ of the synthetic data sets.

Figure $6 C$ provides a sense of the maximum cluster length from all experimental blocks relative to the cluster lengths expected on the basis of random permutations of the data. Here, points to the right of the line of slope 1 (dashed line) had actual maximum cluster lengths that exceeded the $95 \%$ confidence limit provided by the permutation analysis, implying that they reached statistical significance. We conclude that $\sim 31$ and $20 \%$ of experiments in monkeys $\mathrm{G}$ and monkey I yielded significant LIP-pursuit correlations.

In evaluating the frequency of statistical significance that we observed, it is important to remember that the $t$ statistic we used when identifying clusters of positive correlations depends on the number of trials used in the dataset. In general, experiments on monkey $\mathrm{G}$ included more trials than did those on monkey I, probably explaining why we found statistical significance more often in monkey G. For example, a partial correlation of 0.1 when controlling for four variables yields a $t$ statistic of $>2$ when an experiment includes $>402$ trials. The number of usable trials for the two monkeys was $382 \pm 79$ (SD) for $\mathrm{G}$ and $226 \pm 60$ for I. If we had collected still more trials, we might have achieved significance in a larger percentage of our experiments. Our results also would be affected by the choice of 2 as the $t$ threshold for the identification of significance at each time in the LIP-pursuit corrections. The need to choose an arbitrary cluster-forming threshold is an acknowledged problem in suprathreshold cluster tests (Nichols and Holmes, 2002). Note, however, that tests like ours yield good control over the falsepositive rate for the tests no matter the threshold. (Hayasaka and Nichols, 2003). Therefore, our analysis is likely to underestimate the percentage of experiments with real LIP-pursuit correlations.

Trial-by-trial correlations with other oculomotor behaviors

If LIP reflects the salience of a target generally and acts on sensory-motor transformations broadly, then we should find trial-by-trial correlations between the activity of LIP neurons and all aspects of the oculomotor response to a target. We show next equal along the dashed line.

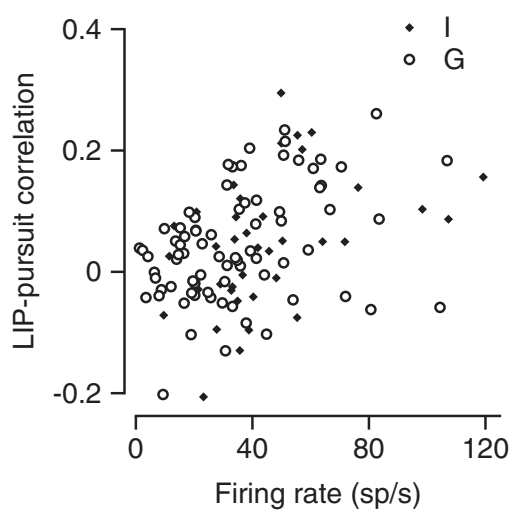

Figure 5. Dependence of the LIP-pursuit correlation on firing rate. The scatter plot shows the LIP-pursuit correlation at the end of the saccade as a function of the average firing rate at the same time. The open and filled symbols show data for monkeys $G$ and $I$.

Figure 6. Statistical reliability of LIP-pursuit correlations for individual experiments. $\boldsymbol{A}$, The cumulative distribution across experments of the maximum cluster length, defined as the longest cluster of contiguous time points where the LIP-pursuit 列

that LIP-pursuit correlations generalize to two other oculomotor behaviors.

LIP-pursuit correlations were present for the small presaccadic pursuit eye velocities that sometimes were present (Fig. 7A, solid lines) even though we designed our experiments to minimize presaccadic pursuit. On average, eye speed exceeded $0.5^{\circ} / \mathrm{s}$ at $143 \mathrm{~ms}$ after the onset of target motion in monkey G. By contrast, eye velocity did not change on average before saccades to stationary targets (Fig. 7A, dashed lines). As expected, the average firing rates of LIP neurons increased well above baseline in the interval around the impending saccade (Fig. $7 B$ ); the response precedes the target movement because of the $40 \mathrm{~ms}$ width of the spike train filter used to estimate firing rate. The difference in the amplitude of the two firing rate traces in Figure $7 B$ probably is related to differences in saccade amplitudes and metrics, rather than to the presence or absence of small smooth eye velocities.

The average across 77 experiments of the LIP-pursuit correlation for presaccadic eye velocity increased after the onset of target motion and reached a positive peak of $\sim 0.1$ for firing rate $80 \mathrm{~ms}$ after the onset of target motion and $45 \mathrm{~ms}$ before the center of the analysis interval for eye velocity (Fig. $7 C$ ). We computed the presaccadic LIP-pursuit correlations for the firing rate in each experiment at different times versus a single measure of presaccadic pursuit strength on each trial: the change in eye velocity from 100 to $150 \mathrm{~ms}$ after the start of target motion. We used a change in eye velocity rather than the absolute eye velocity on 

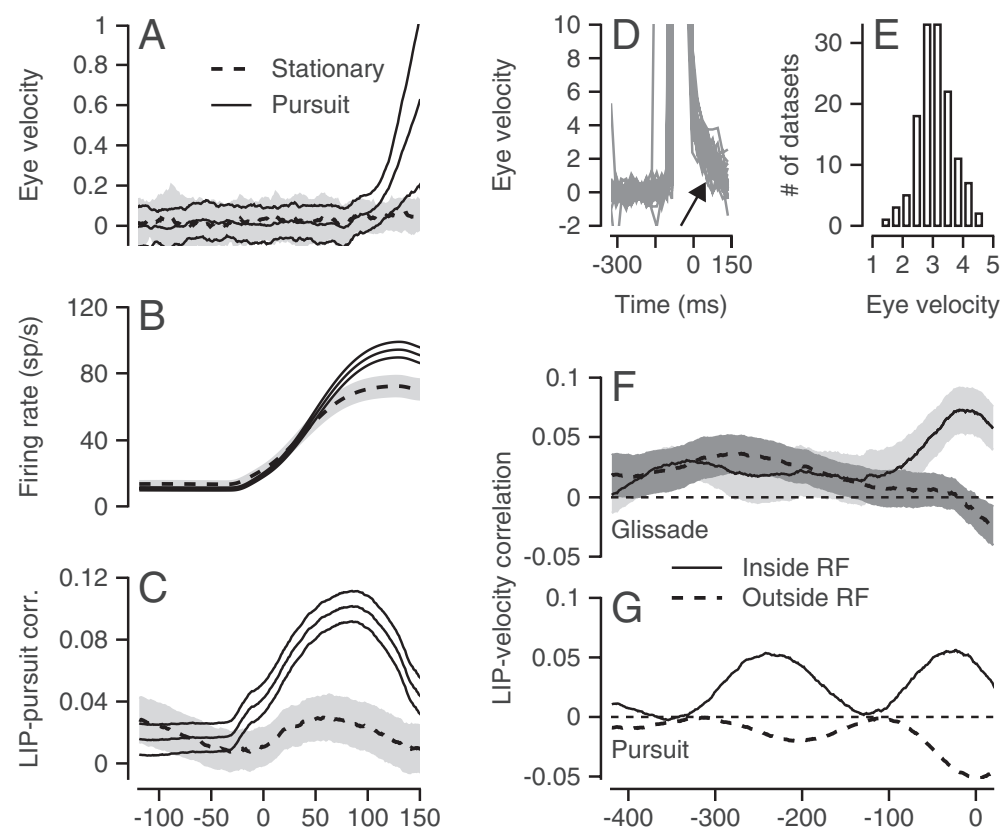

Time relative to target step $(\mathrm{ms})$

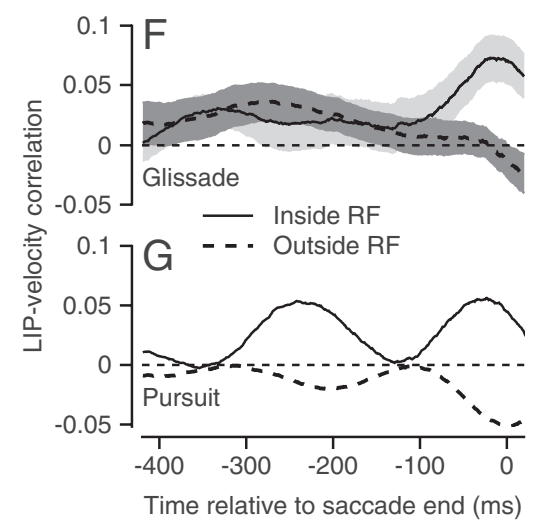

Figure 7. LIP-smooth eye movement correlations for other eye movement behaviors surrounding saccades. $A-C$, Presaccadic pursuit. $\boldsymbol{A}$, Eye velocity as a function of time. $\boldsymbol{B}$, Firing rate as a function of time. $\boldsymbol{C}$, LIP-pursuit correlations as a function of the time used for firing rate in the correlation. All times are relative to the onset of target motion. The continuous and dashed traces show data for moving versus stationary targets inside the receptive field of the neuron under study. Error lines or ribbons show 1 SEM. The change in eye velocity was measured in the interval from 100 to $150 \mathrm{~ms}$ after the onset of target motion. $\mathbf{D}-\boldsymbol{F}$, LIP-glissade correlations. $\boldsymbol{D}$, Sampling of single trial eye velocity traces from one experiment using a stationary target. Traces are aligned on the end of the saccade. The arrow points to the time interval used for the measurement of postsaccadic eye velocity. $\boldsymbol{E}$, Distribution across experiments of the average eye velocity $20-60 \mathrm{~ms}$ after the end of the saccade. $F, G$, Continuous and dashed traces show LIP- eye velocity correlations as a function of the time used to measure firing rate for saccades to stationary targets inside versus outside the receptive field of the neuron under study. The gray error ribbons show 1 SEM. $F$ shows data for glissades, and $\boldsymbol{G}$ replots data for pursuit.

the basis that image motion causes eye acceleration so that the accepted procedure has become to measure eye acceleration or change in eye velocity in presaccadic pursuit (Lisberger and Westbrook, 1985). The average LIP-eye velocity correlations were positive but very small when the monkey made a saccade to a stationary target in the receptive field (Fig. 7C, dashed curve).

We also observed trial-by-trial correlations between the firing rate of LIP neurons and the smooth eye velocity of the "glissades" that frequently follow saccades to stationary targets. Glissades are preprogrammed smooth corrections that come at the end of, and improve the accuracy of, saccades to stationary targets (Fig. 7D, arrow). Unlike postsaccadic pursuit, they do not depend on image motion present before the saccade (Bahill et al., 1978). We collected these data in monkey G, whose glissades comprised eye velocities that ranged from 1 to $5 \%$, measured over the interval from 20 to $60 \mathrm{~ms}$ after the end of the saccade (Fig. 7E).

LIP-glissade correlations (Fig. $7 F$ ) were very similar in magnitude and time course to LIP-pursuit correlations (Fig. 7G). The LIP-glissade correlations (Fig. $7 F$ ) separated for targets within versus outside the receptive field for firing rate $\sim 100 \mathrm{~ms}$ before the end of the saccade (Fig. 7F, solid vs dashed curves), and then peaked for firing rate just before the end of the saccade. As before, we chose a single measure of the postsaccadic eye velocity in the interval from 20 to $60 \mathrm{~ms}$ after the end of the saccade and computed the trial-by-trial correlation of postsaccadic eye velocity in that interval with firing rate as a function of time. The time courses of the LIP-pursuit correlations were similar to the full population for the neurons used to study glissades (Fig. 7G), except that the correlations for targets inside the receptive field of the neuron under study had an earlier positive peak like that seen for the same monkey in Figure $4 B$. We found very little relationship in the values of the peak correlation across smooth eye movement conditions. For the most part, different neurons showed stronger correlations under different conditions.

We did not collect suitable data to perform analysis of LIP-glissade correlations or LIP-presaccadic pursuit correlations in monkey I. Our experiments with visually guided saccades to stationary targets did not contain enough repetitions to perform the correlation analysis for glissades, and this monkey did not generate presaccadic pursuit of large enough amplitude or long enough duration for analysis. Thus, the conclusions from correlations with glissades and presaccadic eye velocity are based on the results from a single monkey and may need to be taken with some caution.

\section{Trial-by-trial correlations of LIP activity with saccade metrics}

As expected if LIP plays a role in determining the saliency of stimuli for many different kinds of movements, LIP-saccade correlations were present for saccade latency, amplitude, and peak velocity, even though less consistent across monkeys and tasks than were the LIP-pursuit correlations. As before, we chose a single measure for each saccade parameter and correlated firing rate over a range of times with that saccade measure. For the correlation with saccade latency, we aligned firing rates with respect to the onset of target motion. For the correlations with saccade amplitude and peak velocity, we aligned firing rates on the end of the saccade. For the latter two variables, we computed partial correlation coefficients, using as control variables the remaining variables taken from saccade latency, saccade amplitude, saccade duration, and peak saccadic speed.

Higher presaccadic firing rates were correlated with shorter saccade latencies (Fig. $8 \mathrm{~A}$ ) for both monkeys and for saccades to moving or stationary targets (Janssen and Shadlen, 2005; Churchland et al., 2008; Kiani et al., 2008). However, the results for saccade amplitude and peak velocity (Fig. $8 B, C$ ) were less consistent. The correlations were fairly strong when monkey $G$ made saccades followed by pursuit of moving targets (bold, continuous curve), but were less persuasive when monkey I made saccades to moving targets or monkey $G$ made saccades to stationary targets.

\section{Discussion}

We have identified multiple relationships between LIP activity and eye movements. The LIP neurons we studied fell into the traditional category of neurons that respond during a delayed saccade task, but we found positive trial-by-trial correlations between variation in firing rate and variation in smooth eye speed either before or after a tracking saccade. The correlations were organized spatially in the sense that they were consistently much 
larger when the target appeared inside the receptive field of the neuron. The LIPpursuit correlations in our data are small on average $(\sim 0.1)$, but in reasonable agreement with the magnitude of neuron-behavior correlations found in other cortical areas. Neuron-pursuit correlations in the smooth eye movement region of the frontal eye field average 0.15 (Schoppik et al., 2008); correlations between trial-by-trial variation in firing rate and saccade latency in LIP range from -0.09 to -0.3 (Janssen and Shadlen, 2005; Churchland et al., 2008; Kiani et al., 2008).

We have observed LIP-pursuit correlations as a "fly-on-the-wall," through analysis of normal variation. We imagine, however, that the correlations reflect a broader role for the activity in LIP in the intentional modulation of smooth eye velocity. LIP-pursuit correlations reached a peak for neural activity that occurred $\sim 60 \mathrm{~ms}$ before the measure we used for pursuit eye velocity. The timing of the effect suggests that either (1) LIP plays a direct and causal role in modulating the gain of visual-motor transmission for pursuit or (2) LIP and pursuit are affected in parallel by external signals that modulate both the saliency of a location in space and the pursuit evoked by target motion through that location. We are intrigued by the causation alternative and by the seemingly promiscuous relationship between LIP firing rate and several eye movement behaviors. However, a blend of these two alternatives seems plausible if multiple brain sites cooperate to create a saliency circuit in which LIP is one node. Noise correlations between neurons within LIP and across cortical areas would allow the discharge of one LIP neuron to be a proxy for the saliency present across the circuit. Indeed, the discharge of an LIP neuron could be correlated with the motor output even if the neuron did not have a direct synaptic effect on the relevant motor circuits.

The spatial specificity of the LIP-pursuit correlations is an important element of our data, but its mechanism is unclear. It is possible that LIP-pursuit correlations were weak for targets outside the receptive field of the neuron under study simply because the firing rates of the neurons were low so that the variance of firing rate also was low. The correlations may be measurable only when firing rate is high for targets inside the receptive field. Then, the spatial specificity of the LIP-pursuit correlations may be secondary to the low firing rates. An alternative is raised by prior findings of examples in which behavior seems to be driven by subsets of a population of neurons. For example, a number of papers have suggested that fine discriminations depend on the subset of neurons that are stimulated on the flanks of their tuning curves (Britten et al., 1996; Purushothaman and Bradley, 2005; Jazayeri and Movshon, 2006; Cohen and Newsome, 2009). Furthermore, the relatively weak correlation between response amplitude and the magnitude of the LIP-pursuit correlations in our data leaves open the possibility that response amplitude is only a small factor. Thus, the low LIP-pursuit correlations for stimuli outside the receptive field could reflect a selective contribution to behavior from the most active neurons in LIP.

One of the most interesting features of neurons in LIP and other cortical areas is the remapping of receptive fields that occurs before the execution of a saccade (Duhamel et al., 1992). We did not study neurons with foveal receptive fields (Ben Hamed et al., 2001), and therefore we were not able to investigate the inter- esting question of whether the remapped component of discharge would show LIP-pursuit correlations. Thus, it remains plausible that the strongest LIP-pursuit correlations would occur for neurons with foveal receptive fields, and that neuron-neuron correlations between peripheral and foveal LIP neurons are responsible for the LIP-pursuit correlations reported here.

Our data do not reveal whether there are parallel fluctuations in smooth eye velocity and the level of firing across the population of neurons in LIP, or whether the profile of modulation fluctuates from trial-to-trial across the LIP population response, as it does in MT (Huang and Lisberger, 2009). These two alternatives reflect different mechanisms. In one, the overall firing rate fluctuates in a correlated way across LIP to reflect a level of arousal or attention that also affects smooth eye velocity. Given the potential role of LIP in attention, we think this interpretation needs to be kept in mind, even though our unpublished efforts have failed so far to establish a strong link between the level of attention to a moving target and the eye velocity of pursuit. In the other, the strength of spatially selectivity in the LIP population response fluctuates across trials and may have a causal role in modulating smooth eye velocity. A different class of experiment will be necessary to resolve the question of how the population response in LIP fluctuates from trial-to-trial in LIP, as well as how the population response is decoded to modulate motor activity.

\section{Role of LIP in pursuit: visual-motor drive versus modulation}

Using more conventional criteria, previous work has suggested that LIP may be involved in the control of pursuit movements. Lynch et al. (1977) identified "visual tracking neurons" in the inferior parietal lobule, but possibly not in LIP. Some neurons in LIP are direction selective for a passively viewed visual stimulus (Fanini and Assad, 2009). Bremmer et al. (1997) found that a surprisingly high percentage (39\%) of the LIP neurons they studied had directional responses during pursuit that resembled those in the smooth eye movement region of the frontal eye fields (Schoppik et al., 2008). Note, however, that these authors did not exclude the possibility that the neurons in their sample simply had receptive fields near the fovea (Ben Hamed et al., 2001). Our sample, in contrast, came from neurons that respond to stationary visual stimuli and before saccades. The small sample of neurons we tested did not have strongly direction-selective responses during pursuit. Thus, our data raise the possibility that neurons in LIP play a role in pursuit even though they do not have conventional responses in relation to the smooth eye velocity of pursuit.

Current models of pursuit eye movements contain parallel pathways with separate functions (Lisberger, 2010). One pathway uses the population response in area MT to estimate target speed 
and direction. It provides signals that drive the initiation of pursuit (Churchland and Lisberger, 2000; Osborne et al., 2005). The other pathway modulates the strength of visual-motor transmission (Schwartz and Lisberger, 1994) through signals that emanate from the smooth eye movement region of the frontal eye field $\left(\mathrm{FEF}_{\mathrm{SEM}}\right)$ (Tanaka and Lisberger, 2001; Nuding et al., 2009). Prior analyses have demonstrated that saccades use the modulation pathway to provide spatially specific control of postsaccadic pursuit eye velocity (Lisberger, 1998; Schoppik and Lisberger, 2006). The consistent finding of LIP-pursuit correlations in relation to the postsaccadic component of pursuit suggests that LIP could play a role in modulation of the strength of visual-motor transformation. In contrast, the dominance of signals related to stationary targets, salience, and saccades in LIP argues against a direct role in driving the initial pursuit response. It is entirely plausible that signals such as those found in LIP could modulate pursuit even though LIP largely lacks signals related to pursuit per se, such as those in the $\mathrm{FEF}_{\mathrm{SEM}}$.

Our data show that small fluctuations in the discharge of LIP neurons are correlated with similar small fluctuations in the strength of pursuit initiation. However, the spatial representation of visual targets in LIP and its seeming function as a salience map (Bisley and Goldberg, 2010) suggest that the real function of LIP in pursuit is to differentiate among multiple moving objects in our visual field. To be effective in stabilizing the motion of an object, pursuit must select one of multiple motions for control of smooth eye movement (Ferrera, 2000; Garbutt and Lisberger, 2006; Shichinohe et al., 2009). The primate brain meets this requirement by adjusting gains of visual-motor transmission spatially to control how strongly pursuit responds to different moving stimuli (Schoppik and Lisberger, 2006). We suggest that the mechanism used by saliency representations to modulate pursuit involves spatially specific control by LIP of the strength of visual-motor transmission from area MT, in effect a form of motor attention. The role of the $\mathrm{FEF}_{\mathrm{SEM}}$ in pursuit gain control (Tanaka and Lisberger, 2001; Nuding et al., 2009) suggests an anatomical substrate for the efferent actions of LIP.

\section{A broader role for LIP in sensory-motor control}

Based on the existence of LIP-glissade correlations as well as LIP-pursuit correlations both before and after the first tracking saccade, we suggest that the same general function of LIP proposed for pursuit also applies to other eye movements and potentially to other motor systems. Thus, one key outcome of our paper is the idea that saliency representations could control sensory-motor behavior through spatially specific modulation of the strength of sensory-motor transmission, as they seem to for pursuit. The pattern of saccade eye movements during hand movements suggests another example of a similar function. As a subject uses the hand to move objects around obstacles from one location to another, saccades point the eyes at critical "via" points just in advance of the hand position (Johansson et al., 2001). This form of eye-hand coordination could be using the saccades to create a salience map in LIP, with the goal of increasing the gain of sensory-motor transmission at specific, critical locations for controlling hand position. We suggest that the efferent actions of LIP may encompass many eye movement behaviors, and possibly many other motor behaviors.

\section{References}

Bahill AT, Hsu FK, Stark L (1978) Glissadic overshoots are due to pulse width errors. Arch Neurol 35:138-142.

Barash S, Bracewell RM, Fogassi L, Gnadt JW, Andersen RA (1991) Saccade- related activity in the lateral intraparietal area. I. Temporal properties; comparison with area 7a. J Neurophysiol 66:1095-1108.

Ben Hamed S, Duhamel JR, Bremmer F, Graf W (2001) Representation of the visual field in the lateral intraparietal area of macaque monkeys: a quantitative receptive field analysis. Exp Brain Res 140:127-144.

Bisley JW, Goldberg ME (2010) Attention, intention, and priority in the parietal lobe. Annu Rev Neurosci 33:1-21.

Bremmer F, Distler C, Hoffmann KP (1997) Eye position effects in monkey cortex. II. Pursuit- and fixation-related activity in posterior parietal areas LIP and 7A. J Neurophysiol 77:962-977.

Britten KH, Newsome WT, Shadlen MN, Celebrini S, Movshon JA (1996) A relationship between behavioral choice and the visual responses of neurons in macaque MT. Vis Neurosci 13:87-100.

Carello CD, Krauzlis RJ (2004) Manipulating intent: evidence for a causal role of the superior colliculus in target selection. Neuron 43:575-583.

Churchland AK, Kiani R, Shadlen MN (2008) Decision-making with multiple alternatives. Nat Neurosci 11:693-702.

Churchland MM, Lisberger SG (2000) Apparent motion produces multiple deficits in smooth pursuit eye movements. J Neurophysiol 84:216-235.

Churchland MM, Chou IH, Lisberger SG (2003) Evidence for object permanence in the smooth pursuit eye movements of monkeys. J Neurophysiol 90:2205-2218.

Cohen MR, Newsome WT (2009) Estimates of the contribution of single neurons to perception depend on timescale and noise correlation. J Neurosci 29:6635-6648.

Duhamel JR, Colby CL, Goldberg ME (1992) The updating of the representation of visual space in parietal cortex by intended eye movements. Science 255:90-92.

Fanini A, Assad JA (2009) Direction selectivity of neurons in the macaque lateral intraparietal area. J Neurophysiol 101:289-305.

Ferrera VP (2000) Task dependent modulation of the sensorimotor transformation for smooth pursuit eye movements. J Neurophysiol 84:2725-2738.

Garbutt S, Lisberger SG (2006) Directional cuing of target choice in human smooth pursuit eye movements. J Neurosci 26:12479-12486.

Gardner JL, Lisberger SG (2001) Linked target selection for saccadic and smooth pursuit eye movements. J Neurosci 21:2075-2084.

Gardner JL, Lisberger SG (2002) Serial linkage of target selection for orienting and tracking eye movements. Nat Neurosci 5:892-899.

Groh JM, Born RT, Newsome WT (1997) How is a sensory map read out? Effects of microstimulation in visual area MT on saccades and smooth pursuit eye movements. J Neurosci 17:4312-4330.

Hayasaka S, Nichols TE (2003) Validating cluster size inference: random field and permutation methods. Neuroimage 20:2343-2356.

Huang X, Lisberger SG (2009) Noise correlations in cortical area MT and their potential impact on trial-by-trial variation in the direction and speed of smooth pursuit eye movements. J Neurophysiol 101:3012-3030.

Janssen P, Shadlen MN (2005) A representation of the hazard rate of elapsed time in macaque area LIP. Nat Neurosci 8:234-241.

Jazayeri M, Movshon JA (2006) Optimal representation of sensory information by neural populations. Nat Neurosci 9:690-696.

Johansson RS, Westling G, Bäckström A, Flanagan JR (2001) Eye-hand coordination in object manipulation. J Neurosci 21:6917-6932.

Kiani R, Hanks TD, Shadlen MN (2008) Bounded integration in parietal cortex underlies decisions even when viewing duration is dictated by the environment. J Neurosci 28:3017-3029.

Krauzlis RJ (2004) Recasting the smooth pursuit eye movement system. J Neurophysiol 91:591-603.

Kusunoki M, Gottlieb J, Goldberg ME (2000) The lateral intraparietal area as a salience map: the representation of abrupt onset, stimulus motion, and task relevance. Vision Res 40:1459-1468.

Lisberger SG (1998) Postsaccadic enhancement of initiation of smooth pursuit eye movements in monkeys. J Neurophysiol 79:1918-1930.

Lisberger SG (2010) Visual guidance of smooth-pursuit eye movements: sensation, action, and what happens in between. Neuron 66:477-491.

Lisberger SG, Westbrook LE (1985) Properties of visual inputs that initiate horizontal smooth pursuit eye movements in monkeys. J Neurosci 5:1662-1673.

Lynch JC, Mountcastle VB, Talbot WH, Yin TC (1977) Parietal lobe mechanisms for directed visual attention. J Neurophysiol 40:362-389.

Newsome WT, Wurtz RH, Dürsteler MR, Mikami A (1985) Deficits in vi- 
sual motion processing following ibotenic acid lesions of the middle temporal visual area of the macaque monkey. J Neurosci 5:825-840.

Nichols TE, Holmes AP (2002) Nonparametric permutation tests for functional neuroimaging: a primer with examples. Hum Brain Mapp 15:1-25.

Nuding U, Kalla R, Muggleton NG, Büttner U, Walsh V, Glasauer S (2009) TMS evidence for smooth pursuit gain control by the frontal eye fields. Cereb Cortex 19:1144-1150.

Osborne LC, Lisberger SG, Bialek W (2005) A sensory source for motor variation. Nature 437:412-416.

Purushothaman G, Bradley DC (2005) Neural population code for fine perceptual decisions in area MT. Nat Neurosci 8:99-106.

Ramachandran R, Lisberger SG (2005) Normal performance and expression of learning in the vestibulo-ocular reflex (VOR) at high frequencies. J Neurophysiol 93:2028-2038.
Schoppik D, Lisberger SG (2006) Saccades exert spatial control of motion processing for smooth pursuit eye movements. J Neurosci 26:7607-7618.

Schoppik D, Nagel KI, Lisberger SG (2008) Cortical mechanisms of smooth eye movements revealed by dynamic covariations of neural and behavioral responses. Neuron 58:248-260.

Schwartz JD, Lisberger SG (1994) Modulation of the level of smooth pursuit activation by initial tracking conditions in monkeys. Vis Neurosci 11:411-424.

Shichinohe N, Akao T, Kurkin S, Fukushima J, Kaneko CR, Fukushima K (2009) Memory and decision making in the frontal cortex during visual motion processing for smooth pursuit eye movements. Neuron 62 : 717-732.

Tanaka M, Lisberger SG (2001) Regulation of the gain of visually guided smooth-pursuit eye movements by frontal cortex. Nature 409:191-194. 